\title{
Designing Energy Materials via Atomic-resolution Microscopy and Spectroscopy
}

Stephen J. Pennycook ${ }^{1,2,3^{*}}$, Xiaoxu Zhao ${ }^{1}$, Jiong $\mathrm{Lu}^{4}$, Wenjie Zang ${ }^{1}$, Haijun $\mathrm{Wu}^{1}$, and John Wang ${ }^{1,2}$

1. Department of Materials Science and Engineering, Faculty of Engineering, National University of Singapore, Singapore.

2. NUS Graduate School for Integrative Sciences and Engineering, Centre for Life Sciences, Singapore.

3. Centre for Advanced 2D Materials, National University of Singapore, Singapore.

4. Department of Chemistry, National University of Singapore, Singapore.

* Corresponding author: steve.pennycook@ nus.edu.sg

In recent years, our sensitivity for imaging and spectroscopy has dramatically improved due to aberration correction, greatly assisting the correlation of atomic-scale structure and bonding to materials' properties. Trial and error materials' development is increasingly being replaced by atomic scale engineering, informed by the powerful combination of microscopy and theoretical calculations. Here we present some recent applications of this approach to catalysts, batteries and thermoelectric materials.

The first direct observation that active catalyst clusters could be as small as dimers or even single atoms was in 1996 [1]. Today, after aberration correction, it has become almost routine to image single atoms and probe their coordination by spectroscopy, greatly aiding the development of single atom catalysts (SACs). Their unique coordination can impart exceptional activity and selectivity, and much effort is ongoing to replace platinum group metals by cheaper, earth abundant metals such as cobalt or nickel. However, to preserve their functionality, SACs must be sufficiently anchored on a support to avoid coalescence into nanoparticles. One such example is the synthesis of graphene-supported cobalt SACs with a tunable high loading using atomic layer deposition [2]. These SACs show exceptional activity and selectivity for the hydrogenation of nitroarenes to azoxy aromatic compounds. Figure 1 shows a highangle annular dark field (HAADF) Z-contrast image where single Co atoms are visible. The electron energy loss (EEL) spectra show that whenever Co is detected, so also is $\mathrm{O}$; it is these proximal $\mathrm{O}$ atoms that expose partially-filled Co-d orbitals, resulting in the excellent catalytic activity.

Another example of excellent activity in a SAC is shown in Figure 2 [3]. Here, cobalt single atoms are anchored in porous nitrogen-doped carbon nanoflake arrays, which are synthesized from a Co-metalorganic-framework (MOF) precursor, followed by acid leaching to remove Co nanoparticles and create extra porosity and active surface area. Interestingly, these SACs show a lower oxygen evolution reaction (OER) overpotential and higher oxygen reduction reaction (ORR) saturation current, than the Co nanoparticle catalysts, showing that Co metal clusters are redundant for both the OER and ORR reactions. The well-dispersed Co single atoms are attached to the carbon network through $\mathrm{N}-\mathrm{Co}$ bonding. The electrocatalyst was then assembled on carbon cloth and used as the air cathode in a solid-state Zn-air battery, achieving good cycling stability (2500 min, 125 cycles) and a high open circuit potential (1.411 V).

Single atom sensitivity is also important for developing thermoelectric materials. Whereas nanostructuring has been well appreciated, recently the key role of interstitials and interstitial clusters on thermal and electrical transport properties has also been elucidated [4]. In piezoelectrics, gradual atomic-scale polarization rotation among co-existing phases has been recently found in lead-free piezoelectrics, [5] a feature that seems common to all high-performance piezoelectric systems at phase boundaries. 


\section{References:}

[1] P Nellist and S Pennycook, Science 274 (1996) p. 413.

[2] H Yan et al., Nature Communications DOI: 10.1038/s41467-018-05754-9 (2018).

[3] W Zang et al., ACS Catal, 8 (2018) p. 8961.

[4] Y Xiao et al., J Am Chem Soc, 139 (2017) p. 18732.

[5] C Zhao et al., J Am Chem Soc, 140 (2018) p.15252.

[6] The authors acknowledge funding from the Ministry of Education, Singapore, under Tier 2 grants 2017 (R-143-000-A06-112), MOE2015-T2-2-094, MOE2017-T2-1-129, MOE2017-T2-2-139 and the National University of Singapore.
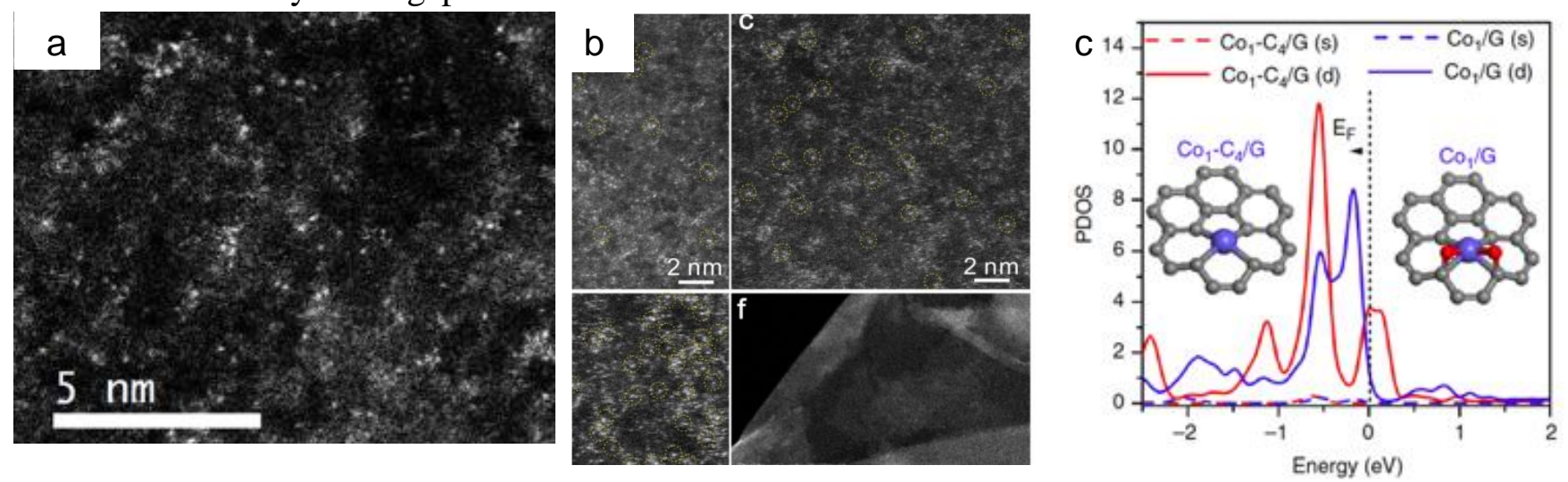

Figure 1. (a) Z-contrast image of Co SAC. (b) EELS showing O associated with the Co atoms. (c) Theoretical simulation of a Co atom (blue ball) with and without $\mathrm{O}$ (red balls), showing their respective partial density of states as blue and red curves. The presence of $\mathrm{O}$ uncovers the Co d orbitals at the Fermi energy. [2]

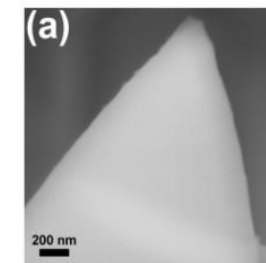

(b)
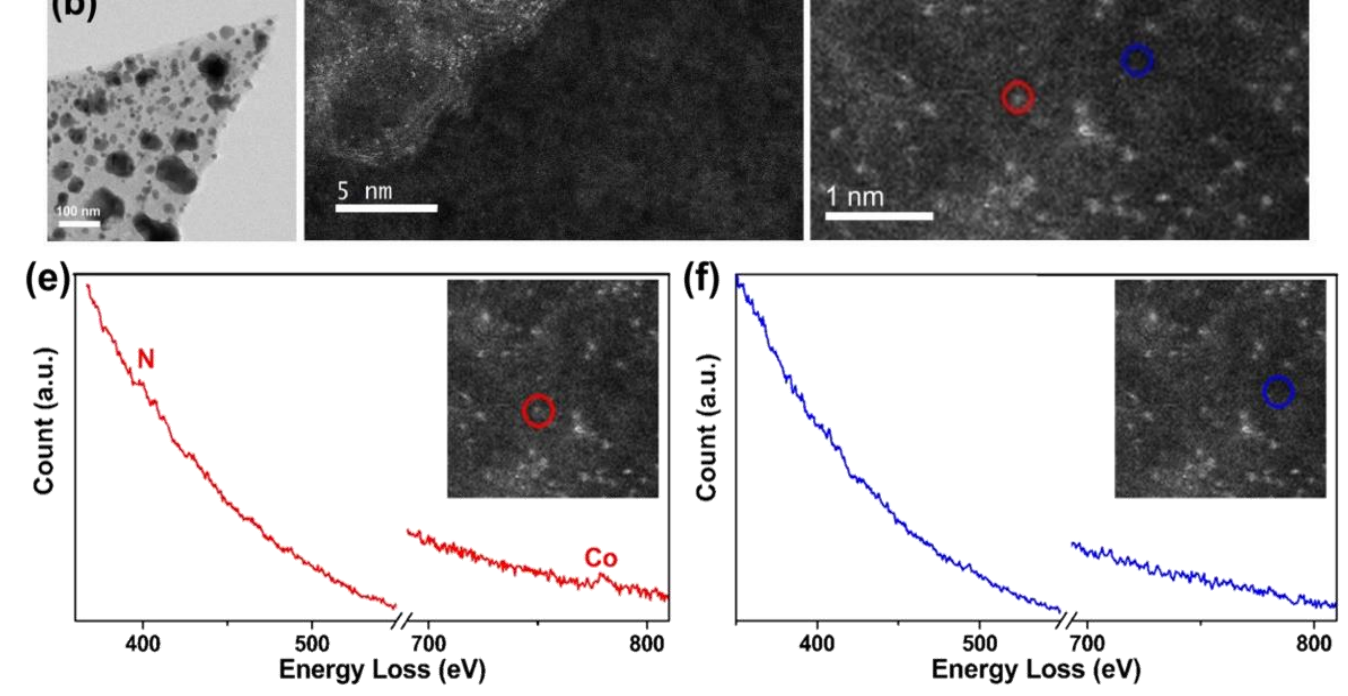

Figure 2. (a) SEM image of a nanoflake. (b) STEM ABF image of a Co nanoparticle catalyst. (c) STEM HAADF image of a Co SAC. Darker regions are where the nanoparticles have been dissolved. (d) HAADF image of Co atoms distributed across the nitrogen-doped carbonaceous support. (e) EEL spectra taken at the bright atom in the red circle in (d) showing both $\mathrm{Co}$ and $\mathrm{N}$ edges. (f) EEL spectra taken at the dark support area in the blue circle in (d) showing neither. [3] 